\title{
Review
}

\section{A Bibliometric Analysis of Dhat Syndrome}

\author{
Sujita Kumar Kar ${ }^{1}$, S. M. Yasir Arafat ${ }^{2, *(D)}$ and Vikas Menon ${ }^{3}$ (D) \\ 1 Department of Psychiatry, King George's Medical University, Lucknow 226003, India; drsujita@gmail.com \\ 2 Department of Psychiatry, Enam Medical College and Hospital, Dhaka 1340, Bangladesh \\ 3 Department of Psychiatry, Jawaharlal Institute of Postgraduate Medical Education and Research (JIPMER), \\ Puducherry 605006, India; drvmenon@gmail.com \\ * Correspondence: arafatdmc62@gmail.com
}

check for

updates

Citation: Kar, S.K.; Arafat, S.M.Y.; Menon, V. A Bibliometric Analysis of Dhat Syndrome. Psychiatry Int. 2021, 2,379-385. https://doi.org/10.3390/ psychiatryint2040029

Academic Editor: Paolo Girardi

Received: 14 September 2021

Accepted: 15 October 2021

Published: 19 October 2021

Publisher's Note: MDPI stays neutral with regard to jurisdictional claims in published maps and institutional affiliations.

Copyright: (c) 2021 by the authors. Licensee MDPI, Basel, Switzerland. This article is an open access article distributed under the terms and conditions of the Creative Commons Attribution (CC BY) license (https:/ / creativecommons.org/licenses/by/ $4.0 /)$.

\begin{abstract}
Dhat syndrome is a culture-bound syndrome, mostly prevailed in South East Asia that has been systematically defined approximately six decades ago. Assessment of the published literature would help to identify the research density as well as the gaps. We aim to do a bibliometric analysis of published global scientific literature on dhat syndrome. The Scopus database was systematically searched by using the keywords "Dhat syndrome", "Semen loss syndrome", "Semen loss anxiety" from inception to 11th August 2021. A total of 89 articles on dhat syndrome were found in the Scopus database. Most of the articles were from India and were published in the Indian Journal of Psychiatry and Asian Journal of Psychiatry. The majority ( $>58 \%$ ) of research on dhat syndrome was published in the last decade. The PGIMER (Chandigarh), AIIMS (New Delhi) \& KGMU (Lucknow) are the leading institutes in dhat syndrome research. The year 2004 had the highest number of total citations ( $n=135$ ), while the year 1975 had the highest number of citations per article (60 citations per article). This bibliometric review identified that despite dhat syndrome is a common condition in the South East Asian countries, research on the condition is limited.
\end{abstract}

Keywords: Dhat syndrome; culture-bound syndrome; bibliometric analysis

\section{Introduction}

Dhat syndrome is described as a culture-bound syndrome, commonly prevalent in South East Asian young males, where the individual's psychological distress is attributed to semen loss [1,2]. Though the term "Dhat syndrome" was introduced nearly six decades back from now (in the 1960s), the entity was recognized as an independent diagnostic category in International Classification of Diseases - tenth edition (ICD-10) and Diagnostic and Statistical Manual of Mental Disorders-4th Edition Text Revision (DSM-IV TR) [1]. Existing literature suggests that dhat syndrome is a common diagnosis in marital and sexual clinics, urology clinics, and dermatology \& venerology clinics [2-5]. Patients of dhat syndrome often present with mood disturbances, somatic symptoms, and disturbances of general well-being, which are often attributed to loss of semen [6-8]. Cultural beliefs about the value of semen and the negative effects of semen loss are to blame for the substantial psychological anguish experienced by dhat syndrome patients. [1,2,9]. As the phenomenology of dhat syndrome has a close resemblance with depression and neurotic spectrum disorders and the diagnostic stability is poor over long-term follow-up, researchers questioned the distinctness of this diagnostic category and suggested that dhat syndrome is a mood disorder with culture-specific expression $[1,10,11]$.

Comorbidities are common with patients with dhat syndrome. For example, sexual dysfunction like premature ejaculation, erectile dysfunction, anxiety, and depression are common comorbidities among patients with dhat syndrome $[6,12,13]$.

Though dhat syndrome has been traditionally managed with antidepressants, anxiolytics, psychoeducation, resolving the sexual myths, cognitive behavior therapy, relaxation exercises, researchers suggested a person-centered approach [14], an integrated approach [1] for dealing with dhat syndrome. 
Research in dhat syndrome evolved over the past several decades focusing on several aspects. Assessment of the published literature would help to identify the research density as well as the gaps. There is no bibliometric analysis of published research on dhat syndrome. On these backgrounds, we aimed to conduct a bibliometric analysis of the global scientific literature on dhat syndrome that has been published to date (11th August 2021) from the time of inception.

\section{Materials and Methods}

The search was done in the Scopus database (www.scopus.com) on 11 August 2012 around 7.35 PM (Dhaka time). The search was carried out from inception to search date using a combination of the following keywords: "Dhat syndrome", "Semen loss syndrome", "Semen loss anxiety", together with the Boolean operator OR.

(TITLE-ABS-KEY ("Dhat syndrome") OR TITLE-ABS-KEY ("Semen loss syndrome") OR TITLE-ABS-KEY ("Semen loss anxiety") AND (EXCLUDE (LANGUAGE, "French") OR EXCLUDE (LANGUAGE, "German”) OR EXCLUDE (LANGUAGE, “Russian”) OR EXCLUDE (LANGUAGE, "Spanish") AND (EXCLUDE (SRCTYPE, "b”).

We included only the journal articles published in the English language. We excluded books, book chapters, conference proceedings, and articles published in other languages. We extracted and analyzed data by Microsoft Excel version 2010 for Windows.

\section{Results}

A search of the SCOPUS database from the time of inception till 11th August 2021 revealed 89 articles (Supplementary Materials), of which 13 were international collaborative work, and two research works were funded. First, we assessed the subject domains under which the dhat syndrome-related results are published. The common subject domains under which dhat syndrome-related articles are published are medicine $(\mathrm{n}=76 ; 85.39 \%)$, followed by psychology $(n=24 ; 26.97 \%)$. The other subject domains under which dhat syndrome was described were Biochemistry, Genetics, and Molecular Biology, Arts and Humanities, Neuroscience, Immunology and Microbiology, Social Sciences, Agricultural and Biological Sciences, Nursing, Pharmacology, Toxicology, and Pharmaceutics.

Similarly, majority of the articles were original research articles $(n=53 ; 59.55 \%)$, followed by correspondences (letters to editors) $(n=19 ; 21.35 \%)$ and review articles (n $=12 ; 13.48 \%)$.

\subsection{Time Trend of Publications}

A total of 89 articles are published between 1975 to 2020 . The majority of the publications $(n=52 ; 58.43 \%)$ were in the past decade (2011-2020). The highest number of articles published in a single year was in 2014 and 2015 ( $n=10$ each). The highest number of total citations has been reported in the year $2004(n=135)$, and the highest citations (60 citations per paper) per paper have been reported in the year 1975. Most numbers of international collaborative work happened in the years 2004 and 2008 ( $n=3$ each) (Table 1).

Table 1. Dhat syndrome research- year-wise output $(n=89)$.

\begin{tabular}{ccccccc}
\hline Year & TP & TC & CPP & \%TP & ICP & ICP\% \\
\hline 2020 & 2 & 0 & 0 & 2.25 & 1 & 7.69 \\
\hline 2018 & 2 & 0 & 0 & 2.25 & 0 & \\
\hline 2017 & 9 & 10 & 1.11 & 10.11 & 0 & \\
\hline 2016 & 8 & 39 & 4.88 & 8.99 & 1 & 7.69 \\
\hline 2015 & 10 & 22 & 2.20 & 11.24 & 1 & 7.69 \\
\hline 2014 & 10 & 75 & 7.5 & 11.24 & 0 & \\
\hline 2013 & 6 & 43 & 7.17 & 6.74 & 1 & 7.69 \\
\hline
\end{tabular}


Table 1. Cont.

\begin{tabular}{lcccccc}
\hline Year & TP & TC & CPP & \% TP & ICP & ICP\% \\
\hline 2012 & 3 & 20 & 6.67 & 3.37 & 0 & \\
\hline 2011 & 2 & 14 & 7 & 2.25 & 0 & \\
\hline 2010 & 2 & 16 & 8 & 2.25 & 0 & \\
\hline 2009 & 2 & 13 & 6.5 & 2.25 & 0 & \\
\hline 2008 & 4 & 60 & 15 & 4.49 & 3 & 7.08 \\
\hline 2007 & 2 & 17 & 8.5 & 2.25 & 0 & \\
\hline 2006 & 2 & 38 & 19 & 2.25 & 0 & \\
\hline 2005 & 2 & 24 & 12 & 2.25 & 1 & \\
\hline 2004 & 6 & 135 & 22.5 & 6.74 & 3 & \\
\hline 2003 & 2 & 7 & 3.5 & 2.25 & 0 & \\
\hline 2001 & 1 & 36 & 36 & 1.12 & 0 & \\
\hline 1999 & 1 & 14 & 14 & 1.12 & 0 & \\
\hline 1998 & 1 & 5 & 5 & 1.12 & 0 & \\
\hline 1996 & 1 & 52 & 52 & 1.12 & 0 & \\
\hline 1995 & 3 & 45 & 15 & 3.37 & 1 & \\
\hline 1991 & 2 & 103 & 51.5 & 2.25 & 0 & \\
\hline 1990 & 1 & 49 & 49 & 1.12 & 0 & \\
\hline 1989 & 2 & 88 & 44 & 2.25 & 0 & \\
\hline 1988 & 1 & 15 & 15 & 1.12 & 0 & \\
\hline 1977 & 1 & 24 & 24 & 1.12 & 0 & \\
\hline 1975 & 1 & 60 & 60 & 1.12 & 1 & \\
\hline
\end{tabular}

TP-Total papers; TC-Total citations; CPP-Citations per paper; ICP-International collaborative paper.

\subsection{Researchers Involved in Dhat Syndrome Research}

There was a total of 160 authors who contributed to publications on dhat syndrome. The total publications among the top ten authors range from 4 to 10. Prof. Ajit Avasthi had the highest number $(n=10)$ of total publications, whereas Prof. Dinesh Bhugra had the highest number of total citations $(\mathrm{n}=135)$, the highest number of total citations per paper, and the highest number of international collaborative work (Table 2).

Table 2. The top ten authors on dhat syndrome research $(n=89)$.

\begin{tabular}{ccccccc}
\hline Author & TP & TC & CPP & \% TP & ICP & ICP\% \\
\hline Avasthi, A. & 10 & 55 & 5.50 & 11.24 & 0 & \\
\hline Grover, S. & 9 & 39 & 4.33 & 10.11 & 0 & \\
\hline Bhatia, M.S. & 7 & 134 & 19.14 & 7.87 & 0 & \\
\hline Prakash, S. & 7 & 13 & 1.86 & 7.87 & 0 & \\
\hline Gupta, S. & 7 & 41 & 5.86 & 7.87 & 1 & 7.69 \\
\hline Kar, S.K. & 7 & 16 & 2.29 & 7.87 & 0 & \\
\hline Balhara, Y.P.S. & 5 & 39 & 7.80 & 5.62 & 0 & \\
\hline Bhugra, D. & 4 & 135 & 33.75 & 4.49 & 4 & \\
\hline Mandal, P. & 4 & 8 & 2.00 & 4.49 & 0 & \\
\hline Tripathi, A. & 4 & 12 & 3.00 & 4.49 & 0 & \\
\hline
\end{tabular}

TP-Total papers; TC-Total citations; CPP-Citations per paper; ICP-International collaborative paper. 


\subsection{Most Productive Organizations in Dhat Syndrome Research}

A total of 86 organizations were involved in the dhat syndrome research. The number of research publications among the top ten organizations ranges from 3 to 14 . The top three organizations involved in dhat syndrome research are Postgraduate Institute of Medical Education \& Research, Chandigarh $(\mathrm{n}=14)$; All India Institute of Medical Sciences, New Delhi $(n=12)$ and King George's Medical University, Lucknow $(n=10)$. The highest number of total citations were from the publications of King's College, London $(n=199)$. The highest number of citations per paper (24.88 citations) and most international collaborations $(\mathrm{n}=6)$ were also from the same institute (Table 3$)$.

Table 3. The top ten institutions on dhat syndrome research $(n=89)$.

\begin{tabular}{|c|c|c|c|c|c|c|}
\hline Institute & TP & TC & CPP & $\% \mathrm{TP}$ & ICP & $\mathrm{ICP} \%$ \\
\hline $\begin{array}{l}\text { Postgraduate Institute of Medical Education \& } \\
\text { Research, Chandigarh }\end{array}$ & 14 & 144 & 10.29 & 15.73 & 2 & 15.38 \\
\hline All India Institute of Medical Sciences, New Delhi & 13 & 69 & 5.31 & 14.61 & 1 & 7.69 \\
\hline King George's Medical University, Lucknow & 10 & 35 & 3.50 & 11.24 & 1 & 7.69 \\
\hline King's College London & 8 & 199 & 24.88 & 8.99 & 6 & 46.15 \\
\hline $\begin{array}{c}\text { Jawaharlal Institute of Postgraduate Medical } \\
\text { Education and Research, Puducherry }\end{array}$ & 7 & 62 & 8.86 & 7.87 & 2 & 15.38 \\
\hline $\begin{array}{l}\text { National Institute of Mental Health and Neuro } \\
\text { Sciences }\end{array}$ & 7 & 54 & 7.71 & 7.87 & 0 & \\
\hline University College of Medical Sciences & 6 & 105 & 17.50 & 6.74 & 0 & \\
\hline Lady Hardinge Medical College & 5 & 63 & 12.60 & 5.62 & 1 & 7.69 \\
\hline JSS Medical College \& Hospital, Mysuru & 4 & 26 & 6.50 & 4.49 & 0 & \\
\hline Sarojini Naidu Medical College & 3 & 21 & 7.00 & 3.37 & 1 & 7.69 \\
\hline
\end{tabular}

TP-Total papers; TC-Total citations; CPP-Citations per paper; ICP-International collaborative paper.

\subsection{Countries with Dhat Syndrome Research}

A total of 14 countries were involved in dhat syndrome research. The total number of publications in the top five countries ranged from 2 to 69 . The maximum ( $\mathrm{n}=69 ; 77.53 \%$ ) publications on dhat syndrome originated from India followed by the United Kingdom $(\mathrm{n}=15 ; 16.85 \%)$. The majority of total citations were from India $(\mathrm{n}=597)$ and the United Kingdom $(n=335)$. The citation per publication was highest for the publication from Sri Lanka (53 citations per paper). The majority of international collaborative work was from India $(\mathrm{n}=10)$ and the United Kingdom $(\mathrm{n}=9)$ (Table 4$)$.

Table 4. The top five countries on dhat syndrome research $(n=89)$.

\begin{tabular}{ccccccc}
\hline Country & TP & TC & CPP & \%TP & ICP & ICP\% \\
\hline India & 69 & 597 & 8.65 & 77.53 & 10 & 76.92 \\
\hline United Kingdom & 15 & 335 & 22.33 & 16.85 & 9 & 69.23 \\
\hline United States & 3 & 65 & 21.67 & 3.37 & 2 & 15.38 \\
\hline Spain & 2 & 18 & 9.00 & 2.25 & 1 & 7.69 \\
\hline Sri Lanka & 2 & 106 & 53.00 & 2.25 & 2 & 15.38
\end{tabular}

TP-Total papers; TC-Total citations; CPP-Citations per paper; ICP-International collaborative paper.

\subsection{Journals Publishing Dhat Syndrome Research}

A total of 43 journals indexed in SCOPUS published dhat syndrome research. The majority of the dhat syndrome-related papers are published in the Indian Journal of Psychiatry $(n=11)$, Asian Journal of Psychiatry $(n=9)$, Indian Journal of Psychological Medicine $(n=7)$. Among the top 10 journals, the number of dhat syndrome-related publications ranges from 2 to 11 . The highest number of total citations are from the 
papers published in the British Journal of Psychiatry $(n=245)$, and the total citations per paper are also maximum (40.83) in the British Journal of Psychiatry. In addition, the most international collaborative works are published in the British Journal of Psychiatry $(n=3)$ (Table 5).

Table 5. The top ten journals on dhat syndrome $(\mathrm{n}=89)$.

\begin{tabular}{ccccccc}
\hline Journal & TP & TC & CPP & \%TP & ICP & ICP\% \\
\hline Indian Journal of Psychiatry & 11 & 64 & 5.82 & 12.36 & 1 & 7.69 \\
\hline Asian Journal of Psychiatry & 9 & 24 & 2.67 & 10.11 & 0 & \\
\hline Indian Journal of Psychological Medicine & 7 & 42 & 6.00 & 7.87 & 0 & \\
\hline British Journal of Psychiatry & 6 & 245 & 40.83 & 6.74 & 3 & 23.08 \\
\hline Journal of Human Reproductive Sciences & 4 & 7 & 1.75 & 4.49 & 0 & \\
\hline Journal of Sexual Medicine & 4 & 58 & 14.50 & 4.49 & 1 & 7.69 \\
\hline Acta Psychiatrica Scandinavica & 2 & 81 & 40.50 & 2.25 & 0 & \\
\hline Indian Journal of Dermatology & 2 & 24 & 12.00 & 2.25 & 0 & \\
\hline Indian Journal of Medical Sciences & 2 & 19 & 9.50 & 2.25 & 0 & \\
\hline International Journal of Social Psychiatry & 2 & 21 & 10.50 & 2.25 & 1 & 7.69 \\
\hline \hline
\end{tabular}

TP-Total papers; TC-Total citations; CPP-Citations per paper; ICP-International collaborative paper.

\subsection{Publishing Houses Involved in Dhat Syndrome Research Publications}

The top five publishing houses that published research on dhat syndrome are Wolter Kluwers, Elsevier, Wiley, Cambridge, and Taylor and Francis. Among these top five publishers, the total number of publications ranges from 8 to 23. The highest number of total publications $(n=23)$ are with Wolter Kluwers publisher. The highest number of total citations $(n=319)$ and citations per paper (39.88) are with the Cambridge publishing group. Most international collaborative works are published by the Wiley $(\mathrm{n}=3)$ and Cambridge $(\mathrm{n}=3)$ publishing groups (Table 6).

Table 6. The five publishers on dhat syndrome research $(n=89)$.

\begin{tabular}{ccccccc}
\hline Publisher & TP & TC & CPP & \% TP & ICP & ICP\% \\
\hline Wolter Kluwers & 23 & 108 & 4.7 & 25.84 & 1 & 7.69 \\
\hline Elsevier & 15 & 96 & 6.4 & 16.85 & 2 & 15.38 \\
\hline Wiley & 10 & 224 & 22.4 & 11.24 & 3 & 23.08 \\
\hline Cambridge & 8 & 319 & 39.88 & 8.99 & 3 & 23.08 \\
\hline Taylor and Francis & 8 & 53 & 6.63 & 8.99 & 1 & 7.69 \\
\hline TP-Total papers; TC-Total citations; CPP-Citations per paper; ICP-International collaborative paper.
\end{tabular}

\section{Discussion}

The present study examined 89 articles available in the SCOPUS database between 1975-2020. These papers received a combined total of 1024 citations averaging 11.51 citations per paper. More than half of the publications were found in the past decade (2011-2020), suggesting greater research focus on Dhat syndrome during this period. However, less than a third of the collaborative research output was produced during the same period (2011-2020). This is an area where further efforts need to be made, given the need for an integrated multi-disciplinary collaborative approach to dhat syndrome management. Such approaches would assist in reducing the stigma associated with a diagnosis of the condition [1].

Authors from India dominated the list of most productive authors on dhat syndrome research. This was also reflected in the countries with maximum research output in this 
condition. India contributed more than three-quarters of total publications and international collaborative papers on dhat syndrome during the period under study. This was followed by the United Kingdom, United States, Spain, and Sri Lanka, with authors from these nations contributing $16.9 \%, 3.4 \%, 2.3 \%$, and $2.3 \%$, respectively. Dhat syndrome is traditionally understood as a culture-bound syndrome, mostly prevalent in Southeast Asia [9]. However, it has been reported from several other geographical regions, including Africa, China, Russia, America, and Europe [2]. Consequently, skewness in research output raises legitimate concerns about the global representativeness of literature on the Dhat syndrome. Interestingly, papers from Sri Lanka had the maximum citations per paper (53.0), suggesting that important work has come out from the country on dhat syndrome.

A glance at the list of most productive institutions on dhat syndrome research reveals two findings: nine of the top ten institutions were from India, and seven of them were public sector health care institutions that contributed more than two-thirds $(69.7 \%)$ of the global research output on dhat syndrome during this period. This suggests a distinct need to expand the research base on dhat syndrome within India too, possibly by incentivizing collaborative research proposals and earmarking research funds for the same. Prior analysis of Indian literature on schizophrenia [15] and bipolar disorder [16] have also noted a similar pattern of research productivity among Indian institutes. Hence, there is a clear need to invest in scaling up research infrastructure across the country. Institutes must formulate policies to incentivize mental health research while the government must prioritize mental health research infrastructure and multi-centric research. It is important to note that such collaborations are needed not only between medical specialties but also between traditional and modern systems of medicine to develop patient-friendly models of care that would have greater acceptability and uptake [17]. Similarly, there is also a need to address the prevalence of sexual myths in the cultures that increases the vulnerability for dhat syndrome. Perhaps, sex education in the family and the school will help in combating sexual myths and preventing the development of dhat syndrome.

The Indian Journal of Psychiatry (12.4\%) followed by the Asian Journal of Psychiatry $(10.1 \%)$ were the two most favored destinations for authors in the field of dhat syndrome. They were followed by the Indian Journal of Psychological Medicine $(7.9 \%)$ and the British Journal of Psychiatry (6.7\%). This may reflect different publishing priorities of journals given the geographical variations in prevalence and burden of dhat syndrome. However, papers published in the British Journal of Psychiatry and Acta Psychiatrica Scandinavica received the highest number of citations with a CPP of 40.8 and 40.5, respectively; they were followed by the Journal of Sexual Medicine (14.5) and the Indian Journal of Dermatology (10.5). This implies that these journals published dhat syndrome papers of greater topical significance, better quality, and higher impact.

A few limitations of the present review must be highlighted. We have only included articles in the SCOPUS database. This means that articles published in journals not listed with SCOPUS would have been excluded. Next, citation metrics of articles in SCOPUS yield more conservative estimates than databases not curated by humans such as google scholar. The articles attributed to individual institutions are not necessarily original articles but reflect all types of research, including correspondences, viewpoints, technical commentaries, editorials, and reviews apart from original research. Additionally, the current bibliographic analysis didn't discuss the epidemiology, theoretical aspect, management (pharma- or nonpharmacological), medical morbidities that could be more clinically relevant. Finally, some Indian journals such as the Indian Journal of Psychiatry and the Indian Journal of Psychological Medicine entered the SCOPUS list in 2009 and 2011, respectively. Hence, publications in these journals before this period did not figure in the bibliometric output. Strengths of the study include using the SCOPUS database to locate published articles; this is a more inclusive database than MEDLINE. Other advantages are the use of several keywords to enhance article pick-up rates and covering a wide period from 1975 till the date of search. 


\section{Conclusions}

This bibliometric review identified that despite dhat syndrome is a common condition in the Southeast Asian countries, research on the condition is limited. There is an extreme lack of international collaboration and funding while studying the condition. There are several Southeast Asian countries where dhat syndrome is not been reported methodically. There is a need for extensive research to understand various aspects of dhat syndrome.

Supplementary Materials: The following are available online at https:/ /www.mdpi.com/article/10 .3390 / psychiatryint2040029/s1, Supplementary file 1: List of articles.

Author Contributions: Conceptualization, S.K.K. and S.M.Y.A. methodology, S.M.Y.A.; software, S.M.Y.A.; validation, S.K.K.; formal analysis, S.M.Y.A.; data curation, S.M.Y.A.; writing-original draft preparation, S.K.K. and VM; writing-review and editing, S.K.K., S.M.Y.A. and V.M.; All authors have read and agreed to the published version of the manuscript.

Funding: This research received no external funding.

Institutional Review Board Statement: Not applicable.

Informed Consent Statement: Not applicable.

Data Availability Statement: All data related to this review is included in the paper.

Conflicts of Interest: The authors declare no conflict of interest.

\section{References}

1. Kar, S.K.; Sarkar, S. Dhat syndrome: Evolution of concept, current understanding, and need of an integrated approach. J. Hum. Reprod. Sci. 2015, 8, 130-134. [CrossRef] [PubMed]

2. Sumathipala, A.; Siribaddana, S.H.; Bhugra, D. Culture-bound syndromes: The story of dhat syndrome. Br. J. Psychiatry 2004, 184, 200-209. [CrossRef]

3. Arafat, S.M.Y.; Ahmed, S. Burden of Misconception in Sexual Health Care Setting: A Cross-Sectional Investigation among the Patients Attending a Psychiatric Sex Clinic of Bangladesh. Psychiatry J. 2017, 2017, 9827083. [CrossRef] [PubMed]

4. Menéndez, V.A.; Fernández-Suárez, J.; Placer, M.; García-Linares, S.; Tarragon, E.L. Dhat syndrome, an emergent condition within urology in Spain. World J. Urol. 2013, 31, 941-945. [CrossRef]

5. Prakash, O. Lessons for postgraduate trainees about Dhat syndrome. Indian J. Psychiatry 2007, 49, 208-210. [CrossRef] [PubMed]

6. Grover, S.A.; Avasthi, S.; Gupta, A.; Dan, R.; Neogi, P.B.; Behere, B.; Lakdawala, A.; Tripathi, K.; Chakraborty, V.; Sinha, M.S.; et al. Phenomenology and beliefs of patients with Dhat syndrome: A nationwide multicentric study. Int. J. Soc. Psychiatry 2016, 62, 57-66. [CrossRef] [PubMed]

7. Prakash, S.P.; Sharan, S.M. A study on phenomenology of Dhat syndrome in men in a general medical setting. Indian J. Psychiatry 2016, 58, 129-141. [CrossRef] [PubMed]

8. Prakash, S.P.; Sharan, S.M. A qualitative study on psychopathology of dhat syndrome in men: Implications for classification of disorders. Asian J. Psychiatry 2018, 35, 79-88. [CrossRef] [PubMed]

9. Prakash, O.S.K.; Kar, T.S.; Sathyanarayana, R. Indian story on semen loss and related Dhat syndrome. Indian J. Psychiatry 2014, 56, 377-382. [PubMed]

10. Mumford, D.B. The "Dhat syndrome": A culturally determined symptom of depression? Acta Psychiatr. Scand. 1996, 94, $163-167$. [CrossRef] [PubMed]

11. Sameer, M.V. Menon, R. Chandrasekaran, Is "Pure" Dhat Syndrome a Stable Diagnostic Entity? A Naturalistic Long Term Follow Up Study from a Tertiary Care Centre. J. Clin. Diagn. Res. 2015, 9, VC01-VC03. [PubMed]

12. Grover, S.; Gupta, S.; Mehra, A.; Avasthi, A. Comorbidity, knowledge and attitude towards sex among patients with Dhat syndrome: A retrospective study. Asian J. Psychiatr. 2015, 17, 50-55. [CrossRef] [PubMed]

13. Rajkumar, R.P. Distinctive clinical features of Dhat syndrome with comorbid sexual dysfunction. Asian J. Psychiatry. 2016, 19, 1-2. [CrossRef] [PubMed]

14. Kar, S.K.; Singh, A. Person Centered Management Approach for the Dhat Syndrome. Int. J. Pers. Centered Med. 2016, 6. [CrossRef]

15. Grover, S.B.M.; Gupta, S.M. Dhawan, Schizophrenia research in India: A scientometricassessmentof India's publications during 1990-2019. Asian J. Psychiatry 2021, 56, 102521. [CrossRef] [PubMed]

16. Grover, S.B.M.; Gupta, S.M. Dhawan, Research on bipolar disorder from India: A bibliometric analysis of papers published during 2000-19. Asian J. Psychiatry 2021, 55, 102532. [CrossRef] [PubMed]

17. Prakash, O.S.K.; Kar, K.S. Dhat Syndrome: A Review and Update. J. Psychosexual Health 2019, 1, 241-245. [CrossRef] 